Ferrata Storti Foundation

\title{
Impact of clinical, cytogenetic, and molecular profiles on long-term survival after transplanta- tion in patients with chronic myelomonocytic leukemia
}

Haematologica 2020

Volume 105(3):652-660

\section{Correspondence: \\ H. JOACHIM DEEG \\ jdeeg@fredhutch.org \\ Received: February 8, 2019. \\ Accepted: July 5, 2019. \\ Pre-published: July 9, 2019.}

doi:10.3324/haematol.2019.218677

Check the online version for the most updated information on this article, online supplements, and information on authorship \& disclosures: www.haematologica.org/content/105/3/652

(C)2020 Ferrata Storti Foundation

Material published in Haematologica is covered by copyright. All rights are reserved to the Ferrata Storti Foundation. Use of published material is allowed under the following terms and conditions:

https://creativecommons.org/licenses/by-nc/4.0/legalcode. Copies of published material are allowed for personal or internal use. Sharing published material for non-commercial purposes is subject to the following conditions:

https://creativecommons.org/licenses/by-nc/4.0/legalcode, sect. 3. Reproducing and sharing published material for commercial purposes is not allowed without permission in writing from the publisher.
Janghee Woo, ${ }^{1,2}$ Dae Ro Choi, ${ }^{1}$ Barry E. Storer, ${ }^{1}$ Cecilia Yeung, ${ }^{1,2}$ Anna B. Halpern, ${ }^{1,2}$ Rachel B. Salit, ${ }^{1,2}$ Mohamed L. Sorror, ${ }^{1,2}$ David W. Woolston, ${ }^{1}$ Tim Monahan, ${ }^{1}$ Bart L. Scott ${ }^{1,2}$ and H. Joachim Deeg ${ }^{1,2}$

${ }^{1}$ Fred Hutchinson Cancer Research Center and ${ }^{2}$ University of Washington School of Medicine, Seattle, WA, USA

\section{ABSTRACT}

hronic myelomonocytic leukemia (CMML) is a heterogeneous group of clonal hematopoietic malignancies with variable clinical and molecular features. We analyzed long-term results of allogeneic hematopoietic cell transplantation in patients with CMML and determined clinical and molecular risk factors associated with outcomes. Data from 129 patients, aged 7-74 (median 55) years, at various stages of the disease and transplanted from related or unrelated donors were analyzed. Using a panel of 75 genes somatic mutations present before hematopoietic cell transplantation were identified In 52 patients. The progression-free survival rate at 10 years was $29 \%$. The major cause of death was relapse (32\%), which was significantly associated with adverse cytogenetics (hazard ratio, 3.77; $P=0.0002$ ), CMML Prognostic Scoring System (hazard ratio, 14.3, $P=0.01$ ), and $\mathrm{MD}$ Anderson prognostic scores (hazard ratio, 9.4; $P=0.005$ ). Mortality was associated with high-risk cytogenetics (hazard ratio, 1.88; $P=0.01$ ) and high Hematopoietic Cell Transplantation Comorbidity Index (score $\geq 4$ : hazard ratio, 1.99; $P=0.01)$. High overall mutation burden ( $\geq 10$ mutations: hazard ratio, $3.4 ; P=0.02$ ), and $\geq 4$ mutated epigenetic regulatory genes (hazard ratio $5.4 ; P=0.003$ ) were linked to relapse. Unsupervised clustering of the correlation matrix revealed distinct high-risk groups with unique associations of mutations and clinical features. CMML with a high mutation burden appeared to be distinct from high-risk groups defined by complex cytogenetics. New transplant strategies must be developed to target specific disease subgroups, stratified by molecular profiling and clinical risk factors.

\section{Introduction}

Chronic myelomonocytic leukemia (CMML) can present either as a myeloproliferative type (MP-CMML) or a myelodysplastic type (MD-CMML), with leukocyte counts of $\geq 13 \times 10^{9} / \mathrm{L}$ and $<13 \times 10^{9} / \mathrm{L}$, respectively, and is therefore classified by the World Health Organization (WHO) as a myelodysplastic/myeloproliferative neoplasm (MDS/MPN). ${ }^{1}$ The clinical course in terms of survival and risk of evolution into acute myeloid leukemia (AML) varies. ${ }^{2}$ Prognosis tends to be worse with MP-CMML than with MD-CMML. ${ }^{3}$ The blast percentage (in blood and bone marrow) has clear prognostic implications. ${ }^{4,5}$ CMML- 0 allows for $<2 \%$ blasts in peripheral blood or $<5 \%$ blasts in the bone marrow or both; CMML- 1 refers to cases with $2 \%$ to $4 \%$ blasts in the peripheral blood or $5 \%$ to $9 \%$ in bone marrow or both; and CMML-2 refers to cases with $5 \%$ to $19 \%$ blasts in peripheral blood, $10 \%$ to $19 \%$ in bone marrow, or the presence of Auer rods.

The highly heterogeneous nature of CMML has led to the development of various prognostic scoring systems in an attempt to assign individual patient risk. ${ }^{6.8}$ These systems have incorporated hematologic indices, cytogenetic abnormalities, and transfusion dependency. More recently, recurrent somatic mutations have been identified in genes associated with signaling pathways (RAS, CBL, CSF3R, and 
JAK2), DNA methylation (DNMT3A, IDH1, IDH2, and TET2), transcription (RUNX1), epigenetic regulation (ASXL1, EZH2, and SETBP1), and splicing (SF3B1, SRSF2, U2AF1, and ZRSR2). ${ }^{9-11}$ TET2 and SRSF2 mutations are the most prevalent in CMML. ${ }^{12,13}$ Patients with MP-CMML have a higher propensity for alterations in signaling pathways, ${ }^{14}$ whereas patients with $\mathrm{MD}$-CMML predominantly have mutations associated with epigenetics. ${ }^{15}$ Recognition of associations between somatic mutations and clinical features has improved the risk stratification of CMML (molecular CMML Prognostic Scoring System, m-CPSS). ${ }^{16}$ Mutations in RUNX1, NRAS, SETBP1, and ASXL1 appear to be associated with unfavorable outcomes. ${ }^{16,17}$

Treatment of CMML with chemotherapy alone only infrequently results in prolonged remission. A randomized trial comparing oral etoposide and hydroxyurea showed superior survival with hydroxyurea. ${ }^{18}$ Treatment with hypomethylating agents results in less toxicity than associated with conventional chemotherapy; but again, remissions tend to be of short duration..$^{19,20}$ The only therapeutic modality with proven curative potential is allogenic hematopoietic cell transplantation (HCT). ${ }^{21-27}$ Published data indicate that the major factors determining long-term relapse-free survival and overall survival are cytogenetic risk category, comorbidities, patient's age and achievement of complete remission..$^{21,25-27}$ In the present study, we analyzed long-term outcomes after allogenic HCT for patients with CMML and, in a subcohort, carried out a comprehensive mutation analysis of 75 genes implicated in myeloid malignancies to define the relationship between somatic mutations and previously established risk factors.

\section{Methods}

\section{Patients}

Between May 1986 and September 2017, 129 patients with CMML underwent HCT at the Fred Hutchinson Cancer Research Center. All provided informed consent for enrollment in investigational protocols and for long-term follow-up as required by the institutional review board of the Center. The characteristics of the patients and their diseases are summarized in Table 1. Patients were 7-74 (median, 55) years of age. The diagnosis and stratification of CMML, and determination of AML transformation were based on WHO 2016 criteria for all cases. ${ }^{1}$ The disease was also risk-categorized by cytogenetics, ${ }^{28}$ the $\mathrm{MD}$ Anderson Prognostic Score (MDAPS), ${ }^{6}$ the CMML-specific Prognostic Scoring System (CPSS), ${ }^{8}$ and the revised International Prognostic Scoring System (IPSS-R). ${ }^{29}$ The HCT Comorbidity Index (HCT-CI) scores were 01 in 35 patients, $2-3$ in 49 patients, and $4-11$ in 45 patients. $^{30}$

\section{Donor and transplant characteristics}

Donor and transplant characteristics are summarized in Table 2. All patients (and donors) were HLA genotyped, following institutional standards. Genotyping was carried out retrospectively in patients transplanted before the routine use of molecular typing. Donors for 42 patients (33\%) were related (38 HLA-identical siblings, 4 HLA-mismatched family members), whereas 87 patients $(67 \%)$ had unrelated donors (68 HLA-matched, 19 HLA mismatched, including 2 cord blood transplants). The stem cell source was bone marrow in $34(26 \%)$ patients, peripheral blood stem cells in 93 (72\%) and cord blood in two. Reduced-intensity conditioning regimens were used in $19 \%$ of patients and high-intensity (myeloablative) regimens in $81 \%$ of patients. Graft-versus-host dis- ease (GvHD) prophylaxis consisted of a calcineurin inhibitorbased regimen in all patients. The severity of any acute and chronic GvHD was assessed and the conditions were treated as described previously. ${ }^{31,32}$

\section{Mutation analysis}

Mutation analysis was performed on DNA from bone marrow mononuclear cells collected prior to transplantation in 52 patients. DNA was extracted using a DNeasy Blood \& Tissue Kit (Qiagen, Valencia, CA, USA), following the manufacturer's protocol. Nextgeneration sequencing libraries were prepared from $400 \mathrm{ng}$ genomic DNA using the Archer VariantPlex Myeloid 75 gene

Table 1. Patient and disease characteristics.

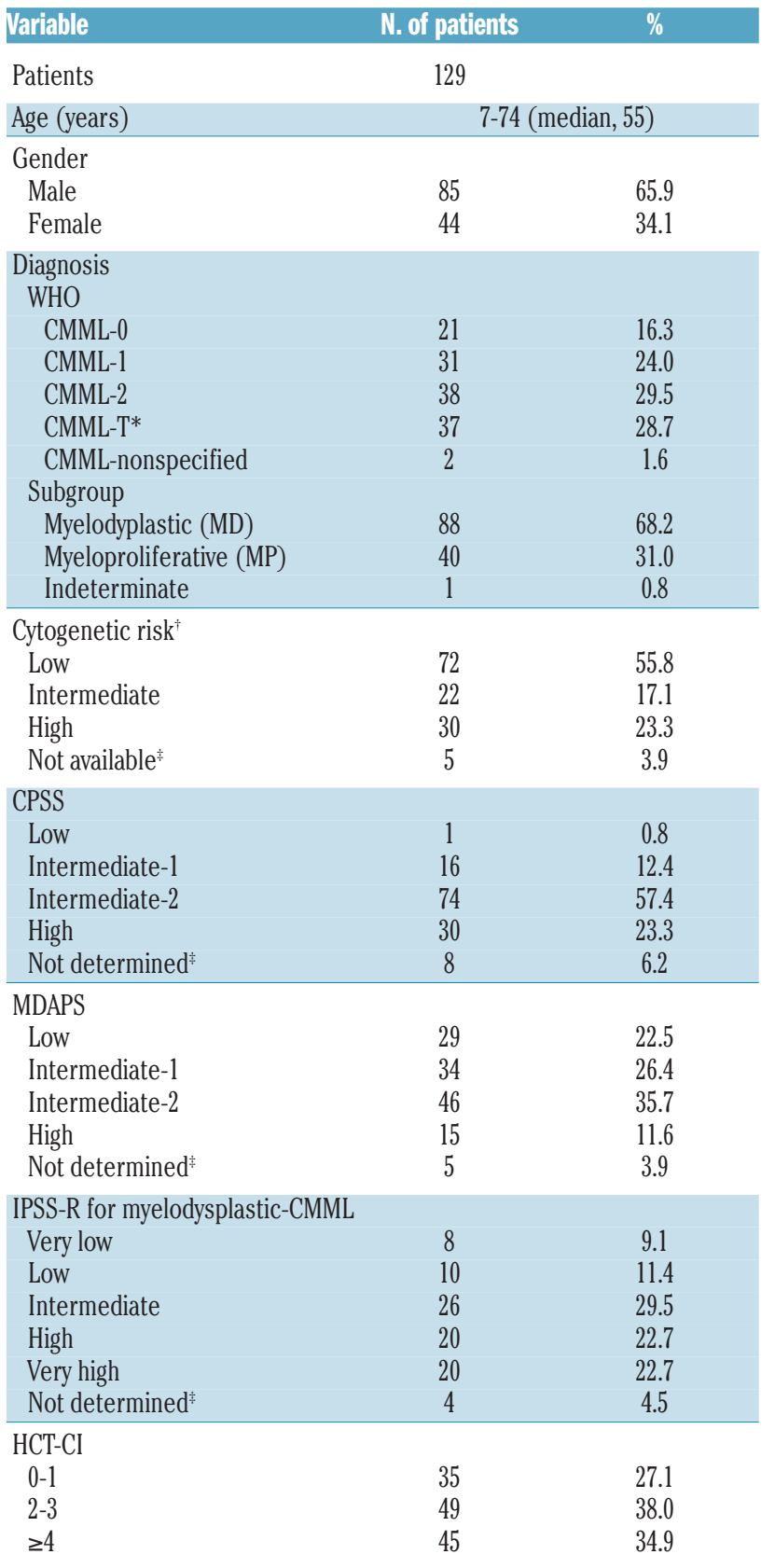

*Progressed to blasts $\geq 20 \%$ with a previous history of CMML. 'According to Such E, et $a l .{ }^{28}{ }^{8}$ Due to failed cell growth in cytogenetic study.WHO:World Health Organization CMML; chronic myelomonocytic leukemia; CPSS: CMML Prognostic Scoring System; MDAPS, MD Anderson Prognostic Score; IPSS-R: Revised International Prognostic Scoring System; HCT-CI: Hematopoietic Cell Transplantation Comorbidity Index. 
panel (ArcherDX, Boulder, CO, USA) following the manufacturer's instructions. We employed highly sensitive and specific targeted sequencing methods, exploiting unique molecular barcodes by anchored multiplex polymerase chain reaction (PCR) chemistry to tag the starting DNA material before PCR amplification. ${ }^{33}$ These methods significantly enhance the sensitivity and specificity of variant detection by filtering out duplicate reads and PCR errors. We followed variant calling procedures as previously reported. ${ }^{34}$ Pairwise associations between mutations and clinical parameters were evaluated by the Fisher exact test and Pearson correlation, corrected for the testing of multiple hypotheses. Hierarchical clustering of associations was performed by in-house scripts in $\mathrm{R} /$ Bioconductor. The Euclidean distance between variables was calculated, and the final partition of variables was obtained by the hclust function in the R package 'stat' with complete linkage as the argument. Details are provided in the Online Supplementary Methods.

\section{Statistical analysis}

Survival was defined as the time from transplantation to death or date of last contact. Relapse-free survival was defined as the time from transplantation to relapse or death from causes other than relapse. Non-relapse mortality (NRM) was defined as death without prior relapse. Estimates of the probability of overall and relapse-free survival were obtained by the Kaplan-Meier method,

Table 2. Donor and transplant characteristics.

\begin{tabular}{|c|c|c|}
\hline Variable & $\begin{array}{l}\mathrm{N} . \\
\text { patients }\end{array}$ & $\%$ \\
\hline \multicolumn{3}{|l|}{ Donor } \\
\hline Related & 42 & 32.6 \\
\hline HLA-matched & 38 & 29.5 \\
\hline HLA-mismatched & 4 & 3.1 \\
\hline Unrelated & 87 & 67.4 \\
\hline HLA-matched & 68 & 52.7 \\
\hline HLA-mismatched* & 19 & 14.7 \\
\hline \multicolumn{3}{|l|}{ Cell source } \\
\hline Bone marrow & 34 & 26.4 \\
\hline Peripheral blood & 93 & 72.1 \\
\hline Cord blood & 2 & 1.6 \\
\hline \multicolumn{3}{|l|}{ Conditioning regimen } \\
\hline \multicolumn{3}{|l|}{ Myeloablative conditioning } \\
\hline $\mathrm{BU}(16 \mathrm{mg} / \mathrm{kg}) / \mathrm{CY}(120 \mathrm{mg} / \mathrm{kg}) \pm \mathrm{ATG}$ & 41 & 31.8 \\
\hline FLU $\left(120 \mathrm{mg} / \mathrm{m}^{2}\right) / \mathrm{BU}(16 \mathrm{mg} / \mathrm{kg})$ & 13 & 10.1 \\
\hline TBI (2 Gy) / I-131-antiCD45 & 12 & 9.3 \\
\hline $\mathrm{BU}(7 \mathrm{mg} / \mathrm{kg}) / \mathrm{TBI}(12 \mathrm{~Gy})$ & 11 & 8.5 \\
\hline BU (7 mg/kg) / CY (50 mg/kg) / TBI (12Gy) & 10 & 7.8 \\
\hline CY $(120 \mathrm{mg} / \mathrm{kg}) / \mathrm{TBI}(14.4$ or $13.2 \mathrm{~Gy})$ & 10 & 7.8 \\
\hline TREO $\left(42 \mathrm{~g} / \mathrm{m}^{2}\right) / \mathrm{FLU}\left(150 \mathrm{mg} / \mathrm{m}^{2}\right) / \mathrm{TBI}(2 \mathrm{~Gy})$ & 9 & 7.0 \\
\hline \multicolumn{3}{|l|}{ Reduced-intensity conditioning } \\
\hline FLU (90 mg/m²) / TBI (2-3 Gy) & 21 & 16.3 \\
\hline Others ${ }^{\dagger}$ & 2 & 1.6 \\
\hline \multicolumn{3}{|l|}{ GvHD prophylaxis } \\
\hline CSP/MTX & 43 & 33.3 \\
\hline CSP/MMF & 36 & 27.9 \\
\hline TAC/MTX & 35 & 27.1 \\
\hline $\mathrm{TAC} / \mathrm{MMF}$ & 6 & 4.7 \\
\hline CSP/MMF/MTX & 2 & 1.6 \\
\hline CSP/others & 7 & 5.4 \\
\hline
\end{tabular}

${ }^{*}$ Includes two cord blood transplants. ${ }^{~} \mathrm{FLU}$ (175 mg/m²) / CY (50 mg/kg) / TBI (3 Gy), FLU $\left(125 \mathrm{mg} / \mathrm{m}^{2}\right) /$ melphalan $\left(140 \mathrm{mg} / \mathrm{m}^{2}\right)$. HLA: human leukocyte antigen; BU: busulfan; CY: cyclophosphamide; ATG: antithymocyte globulin; FLU: fludarabine;TBI: totalbody irradiation; TREO: treosulfan; I-131-antiCD45, iodine-131 monoclonal antibody $\mathrm{BC} 8$; GvHD, graft-versus-host disease; CSP, cyclosporine; MMF, mycophenolate mofetil; TAC, tacrolimus; MTX, methotrexate. and estimates of the probability of relapse, NRM, and GvHD were summarized using cumulative incidence estimates. NRM was considered a competing risk for relapse, and death without chronic $\mathrm{GvHD}$ a competing risk for chronic GvHD. Associations of various factors with the cause-specific hazards of failure for each of these endpoints were assessed using Cox regression. All $P$-values are two-sided and do not incorporate adjustment for multiple comparisons; however, because of the large number of individual mutations considered, we report only associations significant at the 0.01 level of significance, unless the mutation is considered of interest based on prior studies.

\section{Results}

\section{Conventional risk classification}

As shown in Table 1, according to WHO criteria, 21 patients (16\%) had CMML-0, 31 (24\%) had CMML-1, 38 (30\%) had CMML-2, and 37 patients $(29 \%)$ had CMML$\mathrm{T}$ (progressed to a blast count $\geq 20 \%$ with previous history of CMML). In two patients, the staging was inconclusive. In 88 patients $(68 \%)$, the white blood cell count was $<13$ $\times 10^{9} / \mathrm{L}$, thus qualifying as $\mathrm{MD}-\mathrm{CMML}$, and 40 patients $(31 \%)$ had MP-CMML with a white cell count $\geq 13 \times$ $10^{\circ} / \mathrm{L}$. In one patient, the white blood cell count from the pre-HCT period was not available. Among 124 patients with cytogenetic data, $72(56 \%)$ were considered low risk, $22(17 \%)$ intermediate risk, and 30 (23\%) high risk according to the CMML-specific cytogenetic classification. ${ }^{28}$ More than $80 \%$ of patients were "higher" risk according to the CPSS classification (i.e., 30 were high risk and 74 intermediate- 2 risk), while $50 \%$ of patients were "higher" risk according to the MDAPS (i.e., 15 were high risk and 46 intermediate-2 risk).

\section{Engraftment and graft-versus-host disease}

One hundred twenty patients (93\%) achieved sustained engraftment, as defined by absolute neutrophil counts of $\geq 0.5 \times 10^{5} / \mu \mathrm{L}$ for three or more consecutive days. Seven of the remaining nine patients had donor cell engraftment, as determined by chimerism analysis, but died before day 100 without achieving an absolute neutrophil count $\geq 0.5$ $\times 10^{5} / \mu \mathrm{L}$, while two patients died with recurrent CMML. Of the 126 patients evaluable for GvHD (surviving beyond day 28 with donor cell engraftment), acute GvHD of grades II-IV developed in $93(74 \%)$ and grades III-IV in 32 (25\%). Chronic GvHD occurred in 57 patients within 2 years, for a cumulative incidence of $45 \%$.

\section{Relapse}

Relapse or disease progression occurred in 40 patients between 7 and 2,490 (median 154) days after transplantation. The estimated probability of relapse or disease progression was $28 \%$ at 3 years, and $32 \%$ at 10 years (Figure $1 \mathrm{~A}$ ).

\section{Survival}

With a median follow-up of 9.3 (range 0.4-25.2) years, 39 patients are alive while 90 patients have died. The overall survival rates at 3 and 10 years were $38 \%$ and $28 \%$, respectively, while the relapse-free survival rates at the corresponding times were $37 \%$ and $29 \%$ (Figure $1 \mathrm{~A}$ ).

\section{Clinical determinants of post-transplant outcomes}

The results of the univariate analyses are summarized in Table 3. Relapse incidence was significantly increased 
with higher-risk cytogenetics [hazard ratio (HR), 3.77; $95 \%$ confidence interval $(95 \% \mathrm{CI}): 1.9-7.5 ; P=0.0002)]$ (Figure 1B) and the presence of measurable residual disease by cytogenetics at HCT (HR, 2.55; 95\% CI: 1.3-5.0; $P=0.007$ ) (Figure 1C). Classification of CMML by WHO criteria was associated with relapse only with progression to $\geq 20 \%$ blasts (HR, 3.61; 95\% CI: $1.2-10.5 ; P=0.02$ ) (Online Supplementary Figure S1A). Stratification for MDAPS and CPSS showed statistically significant associations with relapse. Specifically, high risk by MDAPS criteria was associated with relapse incidence $[\mathrm{HR}, 5.24$ $(P=0.03), 7.15 \quad(P=0.008)$ and $9.41(P=0.005)$ for intermediate-1 $(n=34)$, intermediate-2 $(n=46)$ and high-risk patients $(n=15)$, respectively] (Figure 1D). Similarly, patients who were classified as high risk by CPSS $(n=30 ; 25 \%)$ had a incidence of relapse than that of lower-risk patients (HR, 14.3; $95 \%$ CI: 1.9-108; $P=0.01$ ) (Figure 1E). Among patients with leukocyte counts $<13 \times 10^{9} / \mathrm{L}(\mathrm{MD}-\mathrm{CMML})$, the very high-risk group, as determined by the IPSS-R $(\mathrm{n}=20)$, was associated with a higher relapse incidence (HR, 7.82; 95\% CI: 1.7-35; P=0.007) (Online Supplementary Figure S1B).

In multivariate analysis the two strongest factors for relapse determined in univariate analysis, cytogenetic risk and MDAPS classification, remained statistically significant (Table 4).

Overall mortality increased with high-risk cytogenetics (HR, 1.88; 95\% CI: 1.2-3.0; $P=0.01$ ) (Figure 1F), the presence of measurable residual disease as determined by cytogenetics (HR, 1.65; 95\% CI: 1.1-2.6; $P=0.02$ ) (Figure $1 \mathrm{G})$, and a high HCT-CI [HCT-CI $\geq 4(\mathrm{n}=45)$ : HR, 1.99; 95\% CI: 1.2-3.4; $P=0.01$ ) (Figure $1 \mathrm{H}$ ), primarily due to increased NRM (HCT-CI $\geq 4$ : HR, 3.39; 95\% CI: 1.5-7.5; $P=0.003$ ) (Figure 1I). Bone marrow blast counts $\geq 20 \%$ at HCT also had a negative impact on survival, although the effect did not reach statistical significance (HR, 1.67; 95\% CI: 0.9-3.2; $P=0.13$ ) (Online Supplementary Figure S1C). Risk stratification by the prognostic scoring systems was generally less predictive of survival, likely due to a high incidence of NRM (Online Supplementary Figure S1D-F). Survival after reduced intensity conditioning was not different from that after myeloablative conditioning (HR, 1.1; 95\% CI: 0.7-1.9; $P=0.71$ ) (Online Supplementary Figure S1G, $H$ ). Pre-transplant therapy and disease status at transplant (complete remission vs. non-complete remission) did not significantly affect overall survival. However, there was a
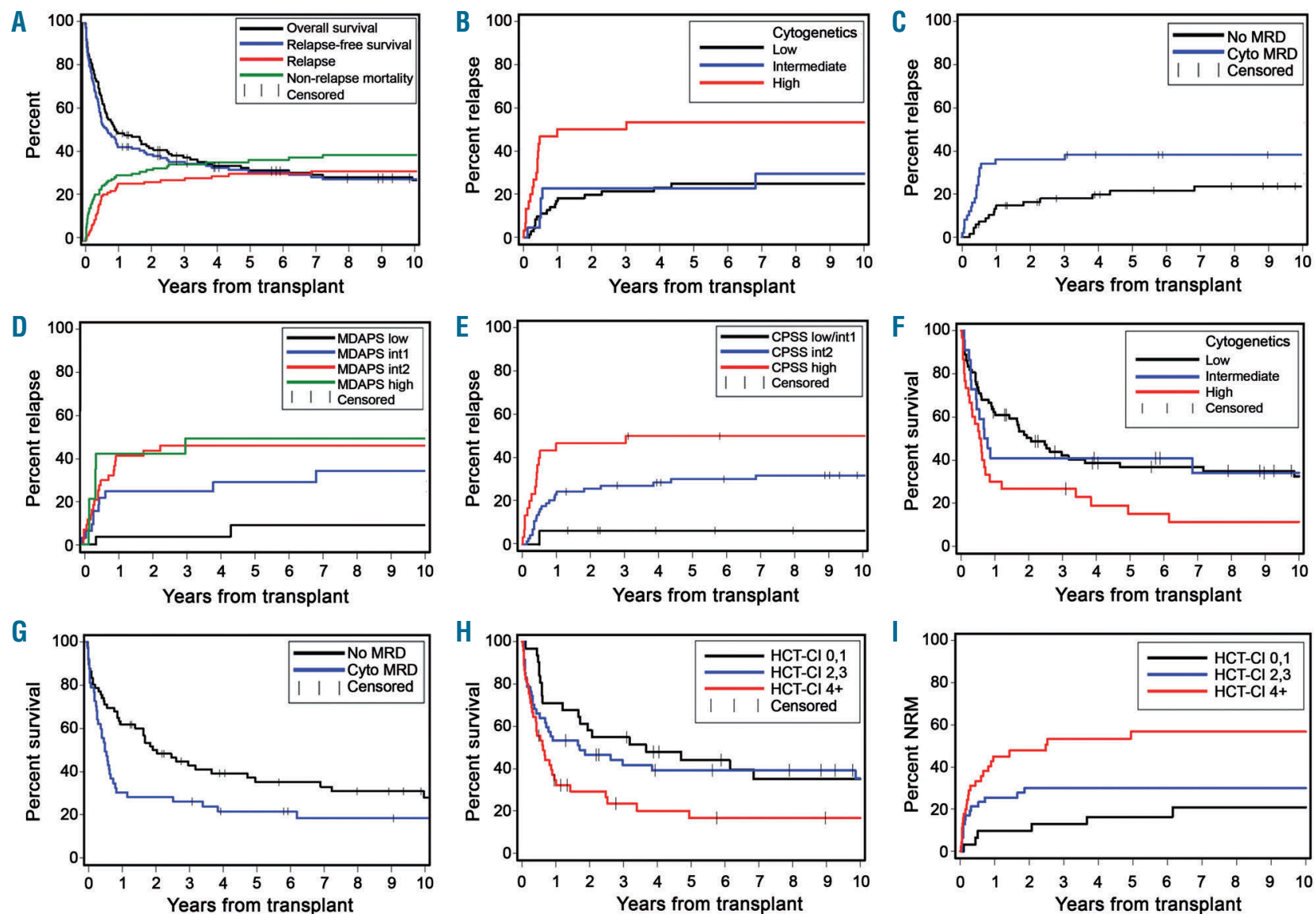

Figure 1. Clinical risk factors associated with relapse and overall mortality/survival in patients with chronic myelomonocytic leukemia following hematopoietic cell transplantation. Overall and relapse-free survival (RFS) and the probabilities of relapse and non-relapse mortality (NRM) are shown for all 129 patients. Tick marks indicate censored patients. (A) Survival, RFS, relapse and NRM for all patients. (B) Relapse by cytogenetic risk. (C) Relapse by measurable residual disease (MRD) at transplantation as indicated by cytogenetics. (D) Relapse by MD Anderson Prognostic Score (MDAPS) and (E) by CMML-specific Prognostic Scoring System (CPSS) risk group. (F) Overall survival dependent upon cytogenetic risk and (G) MRD by cytogenetics. (H) NRM and (I) overall survival by Hematopoietic Cell TransplantationComorbidyt Index (HCT-Cl). 
trend toward higher relapse incidence in patients given pre-HCT intensive cytotoxic chemotherapy and hypomethylating agents, and toward lower relapse incidence with achievement of complete remission at transplant (Table 3). Year of transplant did not have an influence on relapse incidence, overall survival (Table 3), or NRM (for years 2000-2010: HR, 0.7; 95\% CI: 0.38-1.50; $P=0.57$; for years after 2010: HR, 1.05; 95\% CI: 0.49-2.25; $P=0.60)$.

\section{Mutational landscape}

In a subcohort of 52 patients for whom pre-transplant bone marrow samples were available, we carried out a mutational analysis of 75 genes. At least one somatic mutation was identified in 44 of the 52 patients $(85 \%)$ (median number/patient $=5$; range, 0-21) (Online Supplementary Figure S2 and Online Supplementary Tables S1 and S2). The most common mutations were those in ASXL1 (52\%), TET2 (42\%), and SRSF2 (25\%), consistent

Table 3. Univariate regression analyses.

\begin{tabular}{|c|c|c|c|c|c|}
\hline Parameter & & Overall & nortality) & Relaps & sion \\
\hline & N. & HR (95\% Cl) & $P$ & HR (95\% CI) & $P$ \\
\hline Conditioning by intensity & & & & & \\
\hline Myeloablative conditioning & 103 & 1 & & & \\
\hline Reduced-intensity conditioning & 23 & $1.10(0.7-1.9)$ & 0.71 & $1.32(0.6-2.8)$ & 0.46 \\
\hline Age at transplant & & & & & \\
\hline$<55$ years & 62 & 1 & & 1 & \\
\hline $55+$ years & 67 & $1.13(0.7-1.7)$ & 0.57 & $0.95(0.5-1.8)$ & 0.88 \\
\hline CMV serostatus & & & & & \\
\hline Recipient -ve and Donor -ve & 32 & 1 & & 1 & \\
\hline Recipient +ve or Donor +ve & 95 & $1.11(0.7-1.8)$ & 0.67 & $0.92(0.5-1.8)$ & 0.81 \\
\hline Sex match & & & & & \\
\hline Others & 90 & 1 & & 1 & \\
\hline Female to male & 37 & $0.94(0.6-1.5)$ & 0.78 & $0.48(0.2-1.1)$ & 0.07 \\
\hline HCT-CI & & & & & \\
\hline 0,1 & 31 & 1 & & 1 & \\
\hline 2,3 & 47 & $1.10(0.6-1.9)$ & 0.73 & $0.94(0.4-2.0)$ & 0.87 \\
\hline $4+$ & 45 & $1.99(1.2-3.4)$ & 0.01 & $1.08(0.5-2.4)$ & 0.84 \\
\hline Interval (diagnosis to transplant) & & & & & \\
\hline$<6$ months & 29 & 1 & & 1 & \\
\hline 6-18 months & 66 & $1.02(0.6-1.7)$ & 0.95 & $0.97(0.4-2.2)$ & 0.93 \\
\hline$>18$ months & 34 & $1.34(0.7-2.4)$ & 0.33 & $1.72(0.7-4.1)$ & 0.22 \\
\hline Pre-transplant therapy $\dagger$ & & & & & \\
\hline Supportive care & 32 & 1 & & 1 & \\
\hline Low intensity & 34 & $1.01(0.6-1.8)$ & 0.99 & $1.87(0.7-5.1)$ & 0.22 \\
\hline Induction chemo \pm HMA & 38 & $1.31(0.7-2.3)$ & 0.36 & $2.23(0.9-5.9)$ & 0.09 \\
\hline HMA & 25 & $1.37(0.7-2.6)$ & 0.32 & $2.28(0.8-6.4)$ & 0.12 \\
\hline Status at transplant & & & & & \\
\hline Non-complete remission & 64 & 1 & & 1 & \\
\hline Complete remission & 64 & $1.00(0.7-1.5)$ & 0.99 & $0.58(0.3-1.1)$ & 0.1 \\
\hline Individual mutations & & & & & \\
\hline ASXL1 & 27 & $1.4(0.7-2.8)$ & 0.33 & $1.1(0.4-3.0)$ & 0.89 \\
\hline TET2 & 22 & $1.8(0.9-3.6)$ & 0.10 & $1.6(0.6-4.6)$ & 0.40 \\
\hline RUNXI & 9 & $1.1(0.4-2.6)$ & 0.90 & $1.9(0.6-6.1)$ & 0.29 \\
\hline SETBPI & 5 & $0.8(0.2-2.6)$ & 0.67 & $0.6(0.1-4.3)$ & 0.54 \\
\hline$N R A S$ & 6 & $1.9(0.7-5.1)$ & 0.22 & $4.7(1.4-16)$ & 0.03 \\
\hline WT1 & 10 & $4.3(1.7-11)$ & 0.004 & $6.3(1.6-24)$ & 0.01 \\
\hline ATRX & 6 & $4.9(1.8-13)$ & 0.005 & $17.3(4.1-73)$ & 0.0005 \\
\hline Total number of mutations & & & & & \\
\hline$\geq 10$ & 15 & $1.5(0.7-3.2)$ & 0.30 & $3.4(1.2-9.6)$ & 0.02 \\
\hline Functional groups $s^{\ddagger}$ & & & & & \\
\hline Epigenetic & & & & & \\
\hline Trend over $0,1,2,3,4,5$ + mutations & $16,11,12,3,4,6$ & $1.2(1.0-1.5)$ & 0.09 & $1.5(1.1-2.0)$ & 0.02 \\
\hline$\geq 4$ & 10 & $1.5(0.7-3.4)$ & 0.33 & $5.4(1.9-16)$ & 0.003 \\
\hline Tumor suppressor & & & & & \\
\hline Presence & 7 & $2.3(0.9-5.9)$ & 0.09 & $3.1(0.8-11)$ & 0.13 \\
\hline Signaling & & & & & \\
\hline Trend over $0,1,2,3,4,5+$ mutations & $16,11,10,4,4,7$ & $1.1(0.9-1.3)$ & 0.58 & $1.1(0.8-1.5)$ & 0.57 \\
\hline
\end{tabular}

*According to Such E, et al. ${ }^{28}$ Supportive care includes transfusion, treatment with granulocyte colony-stimulating factor and erythropoiesis-stimulating agents. Low intensity treatments include hydroxyurea, lenalidomide, steroids, azathioprine, imatinib, and ruxolitinib. Hypomethylating agents include azacitidine and decitabine. ${ }^{\ddagger}$ According to Online Supplementary Table S1. HR: hazard ratio; 95\% CI: 95\% confidence interval; CMV: cytomegalovirus; HCT-CI: Hematopoietic Cell Transplantation - Comorbidity Index: HMA: hypomethylating agents. 
with previous reports on mutation profiles in patients with CMML. ${ }^{16,17}$ Among the mutations in ASXL1, 67\% were nonsense mutations, while the remainder were either frameshift or stop/gain mutations. Mutations were also frequent in WT1 (27\%), RUNX1 (17\%), DNMT3A (17\%), SMC1A (17\%), EZH2 (12\%), and ATRX (12\%), the high frequency presumably being related to the fact that more than $85 \%$ of our cohort (104 of 129 patients) had intermediate-2 or high-risk disease according to the CPSS (Table 1).

\section{Incorporation of mutations into the overall analysis}

Among mutations with prognostic weight reported in previous studies, ${ }^{16}$ such as ASXL1, NRAS, RUNX1, and SETBP1, only mutations in NRAS ( $\mathrm{n}=6)$ were significantly associated with relapse (HR, 4.7; 95\% CI: 1.4-16; $P=0.03)$. In addition, mutations in ATRX $(\mathrm{n}=6)$ and in the WT1 gene $(\mathrm{n}=10)$ were significantly associated with relapse (HR, 17.3; 95\% CI: 4.1-73; $P=0.0005$; and HR, 6.3; 95\% CI: 1.624; $P=0.01$, respectively) and inferior survival (HR, 4.9; 95\% CI: 1.8-13; $P=0.005$; and HR, 4.3; 95\% CI: 1.7-11; $P=0.004$, respectively).

The impact of mutations was not affected by adjustment in the multivariate analysis, and results remained unchanged.

We then grouped mutations and clinical parameters that co-occurred by unbiased clustering of a correlation matrix across individual mutations and clinical features, including prognostic scoring systems (MDAPS, CPSS, and the CMML-specific cytogenetic classification), leukocyte counts (MP-CMML), and blast counts (Figure 2 and Online Supplementary Figure S3). This approach identified two groups: group 1 mainly included mutations in signaling pathways (e.g. RAS or JAK2) and TP53, associated with high-risk cytogenetics and blasts (Figure $2 \mathrm{~A}$ and Online Supplementary Figure S3A), while group 2 included mutations in epigenetic regulatory genes and splicing factors (Figure 2A). Within this group, mutations in DNMT3A, SETBP1, and EZH2 tended to co-occur, and mutations in TET2 and WT1 were associated with mutations in ATRX. The total number of mutations $[\geq 10$ mutations $(n=15)$ : $\mathrm{HR}, 3.4$; 95\% CI: 1.2-9.6; $P=0.02]$ and greater number of mutations in genes regulating epigenetic processes $[\geq 4$ mutations ( $\mathrm{n}=10)$ : HR, 5.4; 95\% CI: 1.9-16; $P=0.003$ ], but not mutations in signaling pathways and tumor suppressor genes (TP53 and PPM1D), were associated with relapse (Figure 2B, C, Table 3, and Online Supplementary Table S1). When cytogenetics, total number of mutations, established prognostic scoring systems and mutations in epigenetic processes and signaling pathways were considered for their impact on relapse (Figure 2D and Online Supplementary Figure S3B), mutations in epigenetic processes [ $\geq 4$ mutations; odds ratio $(O R)=8.8]$, high blast count $(\geq 20 \% ; O R=4.7)$, high-risk MDAPS $(O R=4.0)$ and high-risk cytogenetics $(\mathrm{OR}=3.2)$ were the major factors contributing to the probability of relapse (details in Online Supplementary Table S3). Pairwise association analysis showed that high blast counts, high-risk by CPSS, and high-risk cytogenetics were closely associated, and mutations in epigenetic regulators tended to occur predominantly in disease classified as high-risk by the MDAPS (Figure 2D and Online Supplementary Table S3). The mCPSS risk model did not allow for further differentiation of risk groups as virtually all patients became classified as intermediate- 2 or high risk. All patients were upgraded to higher risk groups due to their prevalent high-risk mutations. (Online Supplementary Figure S3B). As illustrated in Figure 2E, the delay between diagnosis and HCT was longer (median, 536 days) among patients with higher numbers of mutations than among those with fewer mutations (median delay, 309 days), including epigenetic regulators (522 vs. 344 days), but not mutations in signaling pathways (475 vs. 366 days). These observations were consistent with a previous report on non-transplanted patients showing increasing mutations with longer disease duration. ${ }^{15}$ Overall, the data indicate that molecular annotation uncovered distinct subgroups of CMML that were not distinguished by conventional risk classification. Specifically, a very high-risk group (independent of highrisk cytogenetics and high blast counts) with a long delay to HCT was characterized by a higher number of mutations in epigenetic regulators.

\section{Discussion}

We analyzed the long-term outcomes of allogeneic HCT in 129 patients with CMML in relation to clinical, pathological, and molecular characteristics. The results confirm the curative potential of HCT, since transplanted patients had 3 - and 10 -year relapse-free survival rates of $37 \%$, and $29 \%$, respectively. The outcomes were superior in lowerrisk patients, who had survival probabilities of $40 \%$ to $50 \%$ at 10 years. However, the relapse incidence and NRM rates were high. As in our previous studies ${ }^{21}$ and in reports from other centers, ${ }^{25,26,35}$ high-risk cytogenetics, disease transformation (to CMML-T), and risk classification in scoring systems such as IPSS-R, CPSS and MDAPS, were strongly correlated with post-transplant relapse. As in earlier analyses ${ }^{36}$ there was a suggestion that intensive cytotoxic therapy prior to HCT was associated with inferior outcome, presumably related to the fact that patients considered to be at high risk were more likely to receive induction-type chemotherapy. Nevertheless, it was of note that the intensity of conditioning did not significantly affect overall transplant outcome, although it must be noted that a broad spectrum of conditioning regimens was given to this cohort. Furthermore, as in a previous analy$\mathrm{sis}^{21}$ and in other trials in patients with myeloid malignancies, ${ }^{37}$ patients with high HCT-CI scores had inferior survival, although the probability of relapse was not altered. The source of stem cells did not affect outcome significantly, and results with related and unrelated donors were similar.

The present results indicate that providing HCT at an earlier disease stage and in patients with a low comorbidity burden who have not received cytotoxic therapy results in superior transplant outcomes, as also suggested by others. ${ }^{5}$ These three parameters are likely interrelated as prolonged pre-HCT observation and therapy tend to be associated with the acquisition of new co-morbidities, and likely new mutations, a concept supported by results in our subcohort of patients with mutational data. However, disease characteristics such as high-risk cytogenetics and evidence of measurable residual disease by cytogenetics, still had a profound impact on relapse and survival, even with high-intensity conditioning regimens.

We hypothesize that mutational profiling assists in defining prognosis more narrowly. The analysis of mutations in 75 genes uncovered distinct risk groups defined by 
mutations and disease phenotype which differed for HCT outcomes. In particular, the total number of mutations and more mutations in epigenetic regulators were significantly associated with relapse and with inferior survival. Reminiscent of observations in patients with MPN or MDS, ${ }^{15,38}$ patients with more mutations experienced a higher incidence of relapse, especially when the mutations occurred in genes involved in epigenetic regulation. Those mutations occurred predominantly in patients with dysplastic CMML, as also observed by others. ${ }^{15}$ Of note, however, our unsupervised clustering analysis uncovered a previously unrecognized group of high-risk patients with a higher mutation burden involving epigenetic regulators. This group of patients was not closely associated with high-risk disease as determined by cytogenetics, suggesting independent mechanisms and heterogeneity in the dis-
A

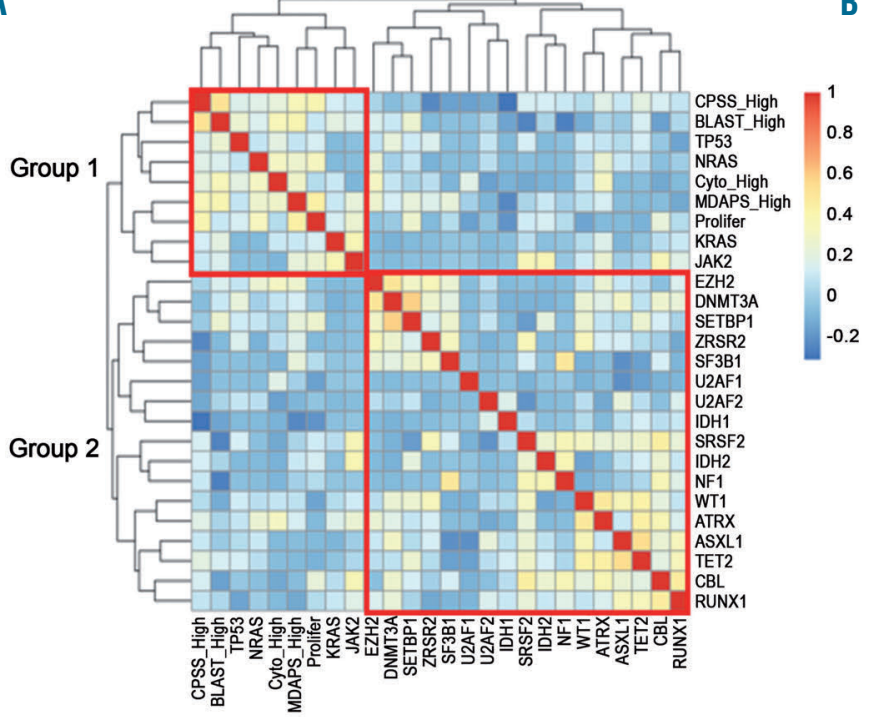

D

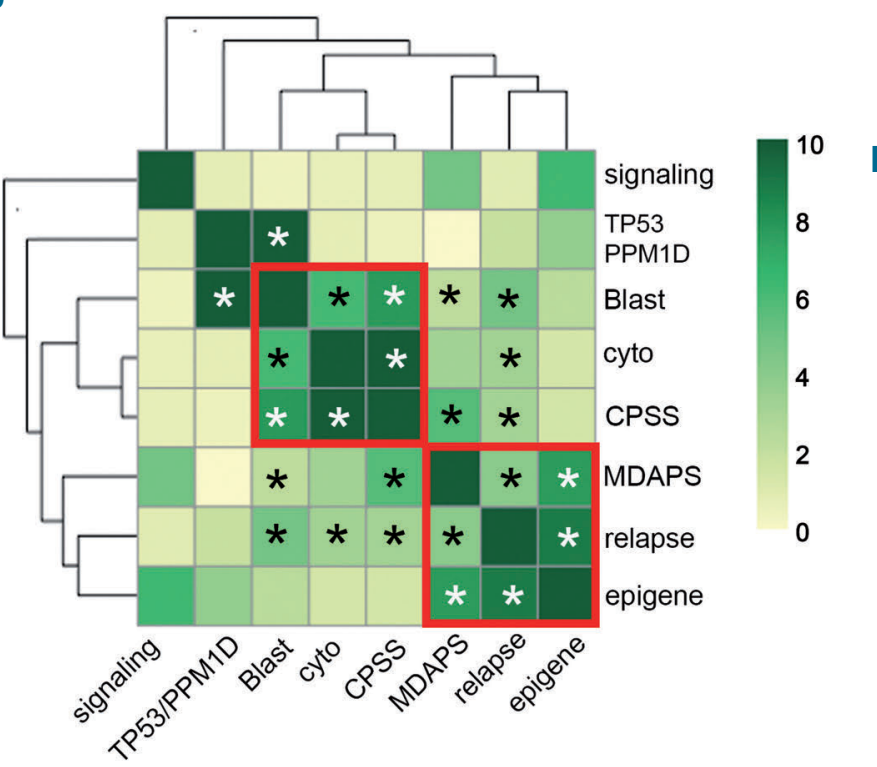

B

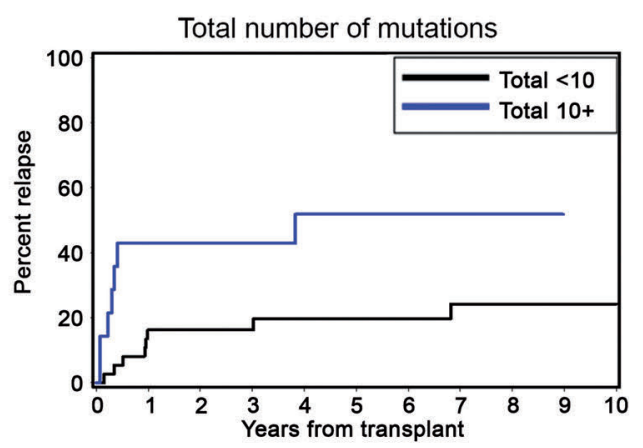

C

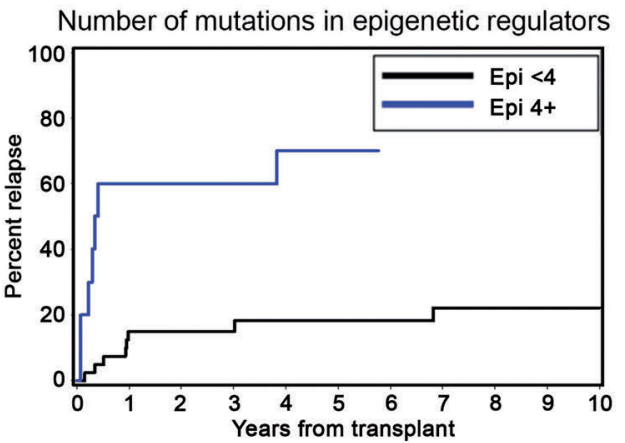

$\mathbf{E}$
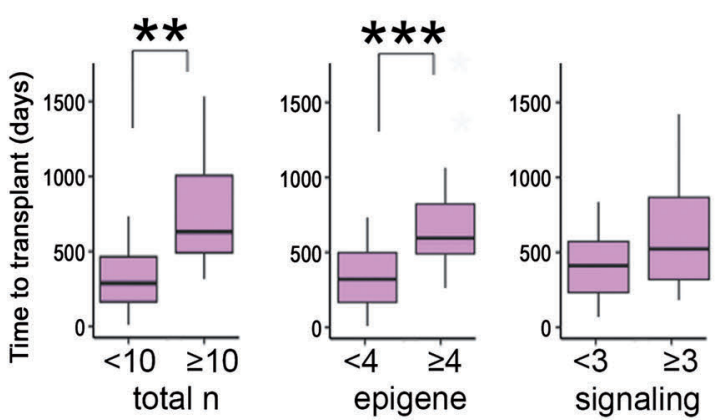

Figure 2. Molecular profiling and risk factors associated with hematopoietic cell transplantation outcomes in patients with chronic myelomonocytic leukemia. (A) Unsupervised hierarchical clustering of a correlation matrix between mutations and clinical parameters. Group 1 consists of mutations in mitotic signaling pathways, myeloproliferative type of chronic myelomonocytic leukemia (CMML) (Prolifer), mutations in TP53, and high-risk disease by cytogenetics and prognostic scoring systems. Group 2 consists of mutations in epigenetic pathways, methylation, and splicing regulation. (B) Increased relapse following hematopoietic cell transplantation in patients with high mutation burden, stratified by total number of mutations $(\geq 10 \mathrm{vs.}<10)$, and $(C)$ stratified by number of mutations in epigenetic regulators $(\geq 4$ vs. <4; classified in Online Supplementary Table S1). (D) Unsupervised clustering of association (odds ratio, corresponding to the scaling bar) between groups of mutations (signaling pathways, epigenetic regulators, and tumor suppressors), high-risk cytogenetics (cyto), risk stratification systems (CPSS, MDAPS) and relapse. $\mathrm{CMML}$ with a high mutation burden in epigenetic regulators (epigene) was distinct from previously recognized high-risk diseases by cytogenetic abnormalities, blast count, and mutations in tumor suppressors (red boxes). (E) Time from diagnosis to transplant. High mutation burden ( $\geq 10$ ), in particular, in epigenetic processes ( $\geq 4$ ), was associated with a longer interval from diagnosis to transplant, consistent with the concept of disease evolution and acquisition of more mutations (in epigenetic but less so in signaling pathways) with disease duration $* P<0.05$ (individual values in Online Supplementary Table S3). **P=0.01, $* * * P=0.03$. 
ease process. Mutations in TP53 were less frequent in patients with CMML ( $<10 \%)$ than previously reported for $\mathrm{MDS}^{39}$ and were not strongly correlated with unfavorable outcomes, underscoring the biological differences between MDS and CMML. The difference may be related to the fact that CMML is infrequently a "secondary disease" (1 of 129 patients in the present series), whereas about $30 \%$ of MDS patients transplanted at our Center present with treatment-related or secondary disease..$^{40}$ The data need to be interpreted with caution, in view of the limited sample size.

The present mutational data suggest a significant association of ATRX and WT1 with post-transplant relapse and overall mortality in patients with CMML. Although the numbers of cases with these mutations were limited, most mutations occurred in important functional protein domains, suggesting that the mutations would be of functional relevance. ${ }^{41}$ Somatic mutations in ATRX are also seen in up to $43 \%$ of patients with MDS with unexplained microcytosis ${ }^{42}$ and in a rare subtype of MDS associated with thalassemia (ATMDS). ${ }^{43}$ Previous studies in CMML have not included ATRX in their mutation panels, ${ }^{16,17}$ and additional investigations are warranted to confirm this association of ATRX mutations with CMML. WT1 mutations are common in patients with high-risk MDS and $\mathrm{AML}^{38}$ where they are associated with relapse. ${ }^{39,44}$ WT1 mutations in the present study occurred in or adjacent to loci that have been shown to be mutated in AML. ${ }^{44}$ Our CMML cohort was predominantly composed of high-risk patients as determined by CPSS and m-CPSS criteria, which, in turn, might be responsible for the prevalence of WT1 mutations and unfavorable transplant outcomes. Although there was a suggestive association of each individual mutation with relapse and survival, the biological impact of these mutations in a limited cohort of CMML patients must be assessed cautiously. Mutations co-occur with other mutations and rarely work as a single dominant factor. Cooperation with other mutations is likely.
Functional data in the right context (e.g., the exact identical mutation at the endogenous locus in hematopoietic cells) are often lacking. The functional consequences of the frequent mutations in ATRX or WT1 will need to be tested in hematopoietic cells to confirm the proposed biological impact of these mutations.

In conclusion, this analysis adds mutational risk factors to previously identified clinical risk factors for post-HCT outcome in patients with CMML, such as comorbidity, cytogenetic risk, and high-risk disease according to the CPSS and MDAPS. Molecular profiling identified distinct high-risk disease groups with high mutation burden, particularly in epigenetic processes that characterized disease entities distinct from the conventional high-risk groups defined by cytogenetics. Of note, the data also show that these high-risk features are only incompletely overcome by HCT, and relapse and NRM rates remain high. This study confirms the clinical and molecular heterogeneity of CMML which significantly affects the outcome following HCT. New transplant strategies that target specific disease subgroups must be developed. Furthermore, early transplantation should be considered for patients with intermediate-risk disease and lower HCT-CI. Vigilant surveillance and early enrollment in clinical trials for post-transplant relapse must be planned for patients with high-risk disease as defined by complex cytogenetics and high mutation burden.

\section{Acknowledgments}

We thank all referring physicians for their continued support and all patients for participating in clinical studies. We are grateful to Gary Schoch for data collection and management, and Helen Crawford for help with manuscript preparation. This work was supported in part by P30 CA015704, P01 CA018029, a Cancer Center New Investigator Support Grant (RBS), a K12 CA076930 (JW), a CTI Biopharma Endowed Fellowship (JW), and an unrestricted grant from Histogenetics (HJD). DRC was supported by a stipend from Hallym University, South Korea.

\section{References}

1. Arber DA, Orazi A, Hasseriian R, et al. The 2016 revision to the World Health Organization classification of myeloid neoplasms and acute leukemia. Blood. 2016;127(20):2391-2405.

2. Germing U, Strupp C, Knipp S, et al. Chronic myelomonocytic leukemia in the light of the WHO proposals. Haematologica. 2007;92(7):974-977.

3. Cervera N, Itzykson R, Coppin E, et al. Gene mutations differently impact the prognosis of the myelodysplastic and myeloproliferative classes of chronic myelomonocytic leukemia. Am J Hematol. 2014;89(6):604-609.

4. Schuler E, Schroeder M, Neukirchen J, et al. Refined medullary blast and white blood cell count based classification of chronic myelomonocytic leukemias. Leuk Res. 2014;38(12):1413-1419.

5. Patnaik MM, Pierola AA, Vallapureddy R, et al. Blast phase chronic myelomonocytic leukemia: Mayo-MDACC collaborative study of 171 cases. Leukemia. 2018;32(11): 2512-2518.
6. Onida F, Kantariian HM, Smith TL, et al. Prognostic factors and scoring systems in chronic myelomonocytic leukemia: a retrospective analysis of 213 patients. Blood. 2002;99(3):840-849.

7. Patnaik MM, Padron E, LaBorde RR, et al Mayo prognostic model for WHO-defined chronic myelomonocytic leukemia: ASXL1 and spliceosome component mutations and outcomes. Leukemia. 2013;27(7):1504-1510.

8. Such E, Germing U, Malcovati L, et al. Development and validation of a prognostic scoring system for patients with chronic myelomonocytic leukemia. Blood. 2013;121(15):3005-3015.

9. Kohlmann A, Grossmann V, Klein HU, et al. Next-generation sequencing technology reveals a characteristic pattern of molecular mutations in $72.8 \%$ of chronic myelomonocytic leukemia by detecting frequent alterations in TET2, CBL, RAS, and RUNX1. J Clin Oncol. 2010;28(24):3858-3865.

10. Papaemmanuil E, Gerstung M, Malcovati L, et al. Clinical and biological implications of driver mutations in myelodysplastic syndromes. Blood. 2013;122(22):3616-3627; quiz 3699.
11. Piazza R, Valletta S, Winkelmann N, et al Recurrent SETBP1 mutations in atypical chronic myeloid leukemia. Nat Genet. 2013;45(1):18-24

12. Kosmider O, Gelsi-Boyer V, Ciudad M, et al. TET2 gene mutation is a frequent and adverse event in chronic myelomonocytic leukemia. Haematologica. 2009;94(12):16761681.

13. Meggendorfer M, Roller A, Haferlach T, et al. SRSF2 mutations in 275 cases with chronic myelomonocytic leukemia (CMML). Blood. 2012;120(15):3080-3088.

14. Ricci C, Fermo E, Corti S, et al. RAS mutations contribute to evolution of chronic myelomonocytic leukemia to the proliferative variant. Clin Cancer Res. 2010;16(8): 2246-2256.

15. Itzykson R, Kosmider O, Renneville A, et al Clonal architecture of chronic myelomonocytic leukemias. Blood. 2013;121(12):21862198.

16. Elena C, Galli A, Such E, et al. Integrating clinical features and genetic lesions in the risk assessment of patients with chronic myelomonocytic leukemia. Blood. 2016;128 (10):1408-1417 
17. Itzykson R, Kosmider O, Renneville A, et al. Prognostic score including gene mutations in chronic myelomonocytic leukemia. J Clin Oncol. 2013;31(19):2428-2436.

18. Wattel E, Guerci A, Hecquet B, et al. A randomized trial of hydroxyurea versus VP16 in adult chronic myelomonocytic leukemia. Groupe Francais des Myelodysplasies and European CMML Group. Blood. 1996;88(7): 2480-2487.

19. Solary E, Itzykson R. How I treat chronic myelomonocytic leukemia. Blood. 2017;130 (2):126-136.

20. Zandberg DP, Huang TY, Ke X, et al. Treatment and outcomes for chronic myelomonocytic leukemia compared to myelodysplastic syndromes in older adults. Haematologica. 2013;98(4):584-590.

21. Eissa H, Gooley TA, Sorror ML, et al. Allogeneic hematopoietic cell transplantation for chronic myelomonocytic leukemia: relapse-free survival is determined by karyotype and comorbidities. Biol Blood Marrow Transplant. 2011;17(6):908-915.

22. Elliott MA, Tefferi A, Hogan WT, et al. Allogeneic stem cell transplantation and donor lymphocyte infusions for chronic myelomonocytic leukemia. Bone Marrow Transplant. 2006;37(11):1003-1008.

23. Itonaga $\mathrm{H}$, Aoki $\mathrm{K}$, Aoki J, et al. Prognostic impact of donor source on allogeneic hematopoietic stem cell transplantation outcomes in adults with chronic myelomonocytic leukemia: a nationwide retrospective analysis in Japan. Biol Blood Marrow Transplant. 2018;24(4):840-848.

24. Kerbauy DM, Chyou F, Gooley T, et al. Allogeneic hematopoietic cell transplantation for chronic myelomonocytic leukemia. Biol Blood Marrow Transplant. 2005;11(9): 713-720.

25. Liu HD, Ahn KW, Hu ZH, et al. Allogeneic hematopoietic cell transplantation for adult chronic myelomonocytic leukemia. Biol Blood Marrow Transplant. 2017;23(5): 767 775.

26. Motohashi K, Fujisawa S, Doki N, et al. Cytogenetic risk stratification may predict allogeneic hematopoietic stem cell transplantation outcomes for chronic myelomonocytic leukemia. Leuk Lymphoma. 2018:59(6):1332-1337.

27. Symeonidis A, van Biezen A, de Wreede L, et al. Achievement of complete remission predicts outcome of allogeneic haematopoietic stem cell transplantation in patients with chronic myelomonocytic leukaemia. A study of the Chronic Malignancies Working Party of the European Group for Blood and Marrow Transplantation. Br J Haematol. 2015;171(2):239-246

28. Such E, Cervera J, Costa D, et al. Cytogenetic risk stratification in chronic myelomonocytic leukemia. Haematologica. 2011;96(3):375-383

29. Greenberg PL, Tuechler H, Schanz J, et al. Revised international prognostic scoring system for myelodysplastic syndromes. Blood. 2012;120(12):2454-2465.

30. Sorror ML. How I assess comorbidities before hematopoietic cell transplantation. Blood. 2013;121(15):2854-2863.

31. Martin P, Nash R, Sanders J, et al. Reproducibility in retrospective grading of acute graft-versus-host disease after allogeneic marrow transplantation. Bone Marrow Transplant. 1998;21(3):273-279.

32. Przepiorka D, Weisdorf D, Martin P, et al. 1994 Consensus Conference on acute GVHD grading. Bone Marrow Transplant. 1995;15(6):825-828.

33. Peng O, Vijaya Satya R, Lewis M, Randad P, Wang Y. Reducing amplification artifacts in high multiplex amplicon sequencing by using molecular barcodes. BMC Genomics. 2015; $16: 589$

34. Woo J, Howard NP, Storer BE, et al. Mutational analysis in serial marrow samples during azacitidine treatment in patients with post-transplant relapse of acute myeloid leukemia or myelodysplastic syndromes. Haematologica. 2017;102(6):e216 e218.

35. de Witte $\mathrm{T}$, Bowen $\mathrm{D}$, Robin $\mathrm{M}$, et al Allogeneic hematopoietic stem cell transplantation for MDS and CMML: recommen- dations from an international expert panel. Blood. 2017;129(13):1753-1762.

36. Zang DY, Deeg HJ, Gooley $\mathrm{T}$, et al Treatment of chronic myelomonocytic leukaemia by allogeneic marrow transplantation. Br J Haematol. 2000;110(1):217-222.

37. Barba P, Ratan R, Cho C, et al. Hematopoietic cell transplantation comorbidity index predicts outcomes in patients with acute myeloid leukemia and myelodysplastic syndromes receiving CD34(+) selected grafts for allogeneic hematopoietic cell transplantation. Bio Blood Marrow Transplant. 2017;23(1):67-74

38. Makishima H, Yoshizato T, Yoshida K, et al Dynamics of clonal evolution in myelodysplastic syndromes. Nat Genet. 2017;49(2): 204-212.

39. Lindsley RC, Saber W, Mar BG, et al Prognostic mutations in myelodysplastic syndrome after stem-cell transplantation. $\mathrm{N}$ Engl J Med. 2017;376(6):536-547.

40. Deeg HJ, Scott BL, Fang M, et al. Five-group cytogenetic risk classification, monosomal karyotype, and outcome after hematopoietic cell transplantation for MDS or acute leukemia evolving from MDS. Blood. 2012;120(7):1398-1408.

41. Shi J, Wang E, Milazzo JP, Wang Z, Kinney JB, Vakoc CR. Discovery of cancer drug targets by CRISPR-Cas9 screening of protein domains. Nat Biotechnol. 2015;33(6):661667.

42. Herbaux C, Duployez N, Badens C, et al Incidence of ATRX mutations in myelodysplastic syndromes, the value of microcytosis. Am J Hematol. 2015;90(8):737-738.

43. Steensma DP, Higgs DR, Fisher CA, Gibbons RJ. Acquired somatic ATRX mutations in myelodysplastic syndrome associated with alpha thalassemia (ATMDS) convey a more severe hematologic phenotype than germline ATRX mutations. Blood. 2004;103(6):2019-2026.

44. Rampal R, Figueroa ME. Wilms tumor 1 mutations in the pathogenesis of acute myeloid leukemia. Haematologica. 2016;101(6):672-679. 